\title{
Modeling the effect of film-pore coupled transport on composite Forward Osmosis membrane performance
}

\author{
Seetha S Manickam ${ }^{1}$, Guy Z. Ramon ${ }^{2 *}$, Jeffrey R. McCutcheon ${ }^{1}$ \\ ${ }^{1}$ Department of Chemical \& Biomolecular Engineering, \\ Center for Environmental Sciences and Engineering, \\ University of Connecticut, Storrs, CT, USA \\ ${ }^{2}$ Department of Civil \& Environmental Engineering, \\ Technion - Israel Institute of Technology, Haifa, Israel
}

*corresponding author

Telephone: +972-4-8292580

Email: ramong@technion.ac.il 


\begin{abstract}
The methods used to quantify membrane structural contributions to transport resistance in engineered osmosis (EO) processes contain inherent assumptions and inaccuracies. Consequently, incomplete information is available on the actual effects of the support layer and overall composite membrane characteristics, on EO performance. In this work, the effects of support properties such as pore radius, porosity and thickness on membrane transport are studied using a 2D numerical model that accounts for coupled transport of water and solute within both the selective thin-film as well as the support pores. The results indicate that reducing support thickness by half enhanced performance to a greater extent $(\sim 40$ $50 \%$ increase in net driving force recovered) than did either doubling porosity (22-34\% increase in net driving force recovered) or increasing pore radius by two orders of magnitude (15-28\% increase in net driving force recovered). Further, the effect of the support pore radius, not included in the commonly employed structural parameter equation (which includes porosity, tortuosity and thickness), was found to impact performance. It was seen that the individual geometrical features comprising the structural parameter affect performance to varying degrees and thus future membrane design could benefit from tuning these parameters accordingly, so as to achieve optimal performance.
\end{abstract}

Keywords: forward osmosis, pressure retarded osmosis, transport models, numerical simulation, internal concentration polarization 


\section{Introduction}

Engineered osmosis (EO) is an emerging technology platform comprising a number of membrane-based processes including forward osmosis (FO), pressure-retarded osmosis (PRO), direct osmotic dilution and direct osmotic concentration, which can be used for water treatment [1], power production [2, 3], emergency relief scenarios [4] and dewatering [1], respectively. These technologies rely on the osmotic pressure difference between a concentrated draw solution and a relatively dilute feed solution. Specifically, the water flux attained by EO membranes, typically thin film composites (TFC) is dependent on the osmotic pressure gradient realized over the selective layer of the TFC membrane. The membrane support layer, however, poses a resistance to draw (in FO) and feed (in PRO) solute mass transport thus dramatically reducing the available driving force. This phenomenon is referred to as internal concentration polarization (ICP) and is a major limitation in commercializing EO processes [5]. The EO community commonly uses the intrinsic structural parameter, $S_{\text {int }}$, [6] as a metric to denote the influence of the average bulk structural properties on the severity of ICP. $S_{\text {int }}$ is defined as

$$
S_{\text {int }}=\frac{t \tau}{\varepsilon}
$$

where $t$ is the thickness, $\tau$ is the tortuosity, and $\varepsilon$ is the porosity of the membrane structure. Quite obviously, membranes with a low $\mathrm{S}_{\text {int }}$ value are preferred in order to reduce structural resistances to transport (by decreasing the effective diffusion path length for solute diffusion) thus reducing the severity of ICP. To this end, most EO membrane developers have focused on optimizing the support layer characteristics by individually manipulating the different structural metrics $\left(t, \tau\right.$ or $\varepsilon$ ) to reduce $S_{\text {int }}[7]$. However, post-membrane fabrication, characterizing these metrics and hence the overall value of $S_{\text {int }}$, is a challenge for such soft materials. A common alternative to measuring $S_{\text {int }}$ has been to estimate an effective structural parameter, $S_{\text {eff, }}$ from osmotic water flux measurements. This is done with the use of semiempirical models derived from a 1D advection-diffusion equation within the support, with boundary conditions that reflect transport through the selective thin film and 'external' advection using film theory 
[5]. In order to maintain the simplicity required for an analytical solution, a number of assumptions are implicit in these models (e.g., constant solution properties, membrane transport coefficients, film theory). Consequently, when a fitting procedure is applied to experimental data, all possible sources of model inaccuracies conspire to create artificial deviations in the 'effective' structure factor. For example, in a recent attempt to standardize FO membrane testing, it was observed that even when identical testing conditions were used by different research groups to evaluate two sets of membranes obtained from the same manufacturing batch, the $S_{\text {eff }}$ values varied non-negligibly [8]. Further, significant deviations between the intrinsic and effective structural parameters have been observed in both previous studies by these authors as well as others [9], the latter also implicating the importance of solution-membrane interactions such as charge uptake and swelling, which induce structural changes and hence alter membrane properties - once again, such effects will be absorbed by the adjustable parameter. The main idea behind the simple, analytical model is that there is a mass transfer resistance in the support, however, any attempt to link this to microscopic membrane attributes is crude at best. Thus the use of the effective structural parameter as a quantitative measure of membrane structure can be misleading and, furthermore, comparisons between the effectiveness of different membrane structures in an EO process on such basis, as is quite commonly done in the literature, would be inherently flawed.

It is thus imperative to find alternate approaches to reasonably evaluate the influence of structural parameters and assess their effect on composite membrane transport and ultimately, performance. Perhaps, the three structural metrics $(t, \tau$ and $\varepsilon$ ) impact membrane transport to varying degrees and need to be weighted differently. Apart from these three average parameters there also exist other parameters that might influence how the structure affects flux performance, such as pore radius, pore-pore spacing (porosity), pore geometry etc. Furthermore, limitations of the structural parameter concept in deducing structural resistance to transport also means that there are currently no methods to reliably compare the new and novel EO membranes being made.

In this work, a two-dimensional numerical model is formulated in order to study the impact of 
geometrical variables on overall composite membrane transport. Such a modeling approach serves as a relatively rapid method of evaluating the effect of different parameters on a given process and has, in fact, previously been used to study the impact of support layer properties on reverse osmosis (RO) membrane transport [10-14], and was corroborated experimentally [15]. Here, a model geometry similar to that devised by Ramon et al. [10] is used and a mathematical model describing the coupled transport in both the selective layer (film) and the porous support is developed, along with appropriate boundary conditions. The influence of the support thickness, porosity and pore size, as well as the effect of varying draw and feed concentrations, on pore transport are studied. The parameters considered are the "average" geometrical values (for e.g. average porosity of the entire membrane structure) and thus variations of these values over the depth of the membrane are not taken into account. The impacts of varying these geometrical parameters on the severity of ICP have been examined and the possibility of varying relative importance of support porosity versus thickness on membrane performance was explored.

\section{Model formulation}

The purpose of the developed model was to assess, quantitatively, the impact of the support structure, namely pore radius, porosity and thickness, on the transport across the membrane. Additionally, the effect of feed and draw concentrations has also been examined. A schematic of the 2D model geometry used in the numerical simulations is shown in Fig.1. An axi-symmetric, periodic geometry was used in order to reduce the computation times, where only a unit cell is solved, representing a larger scale system composed of many repeating units; it has been previously shown that a three-dimensional, square-array structure yields results qualitatively identical and quantitatively only slightly different from an axisymmetric, periodic geometry [10]. The model is thus an idealized version of a complex reality, since several features are not accounted for within the simplified geometrical framework. These include the detailed features of the thin-film morphology (discussed, for example, in refs $[11,14]$ ) as well as the intricate details of the support pore structure. These may have three-dimensional attributes that vary with the depth of the support, e.g. connectivity, tortuosity as well as the actual pore size. However, the main 
purpose of the current study is to consider the transport at the film-pore interface; furthermore, the simplified geometry facilitates comparison with available experimental data. Therefore, the model presented herein may be considered as a first step towards understanding the coupled transport occurring in the polymeric thin film and the support pore-space.

\section{FIGURE 1}

\subsection{Film transport}

In the model framework, it is assumed that the film is perfectly selective (i.e. 100\% solute rejection). This is, of course, not representative of any commercial membrane where some salt transport is always present; however, salt leakage represents a detail which is not expected to impact the model, qualitatively, as far as the geometrical restrictions of the film-pore interface are concerned. It is further assumed that transport through the thin-film is diffusive, driven by gradients in the chemical potential of the diffusing species (water) across the film. Hereafter, we represent the chemical potential as a concentration of water. The steady state concentration field within the film is governed by the 2D Laplace equation,

$$
\frac{1}{r} \frac{\partial}{\partial r}\left(r \frac{\partial c_{w}}{\partial r}\right)+\frac{\partial^{2} c_{w}}{\partial z^{2}}=0
$$

where $\mathrm{c}_{\mathrm{w}}$ is the molar concentration of water and $\mathrm{r}$ and $\mathrm{z}$ represent the radial and axial (along the thickness of the membrane) coordinates in the system (see schematic drawing in Fig. 1). The following boundary conditions are imposed in solving the above equation:

At the film-draw interface, $c_{w}=c_{w D}$

Here $c_{w D}$ is the molar concentration of water in the bulk draw solution. At the film-pore interface, we set the osmotic water flux to be equal to the diffusive water flux, i.e., 


$$
A \Delta \pi=\frac{D_{w}}{\rho_{w} M_{w}} \frac{d c_{w}}{d z}
$$

or, effectively,

$$
A\left(\pi_{D}-\pi_{F}\right)=\frac{D_{w}}{\rho_{w} M_{w}} \frac{c_{w, D}-c_{w, f-s}}{t_{f}}
$$

In the above equations $A$ is the water permeance in the film, $\pi$ is the osmotic pressure, subscripts $D$ and $F$ correspond to the draw and feed solutions, $D_{w}$ is the diffusivity of water in the film, $\rho_{w}$ is the density of water, $M_{w}$ is the molecular weight of water and $t_{f}$ is the thickness of the selective film. The membrane

permeance, $A$, was set to be $2.7 \cdot 10^{-13} \mathrm{~m} / \mathrm{s} / \mathrm{Pa}$ to make it fairly consistent with the use of experimentally determined $A$ values of TFC membranes with a geometry similar to the one in Fig. 1 [6]. While this is not a rigorous way of calculating the water permeance, we resort to it due to a lack of reliable data for transport in the isolated thin-film. $D_{w}$ was set to be $0.7 \cdot 10^{-9} \mathrm{~m}^{2} / \mathrm{s}$ based on studies on diffusivity of water in polyamide films [16-20]. The only unknown in Eqn. 4 is the concentration of water at the film-support interface, which is found from the solution of the model equations.

\subsection{Pore transport}

In the support domain, transport is assumed to occur only within the pore and the support matrix is assumed to be impermeable. This is a fair assumption since even in the case of hydrophilic support materials the rate of transport through fluid-filled pores will far exceed that through the solid polymer. In the pore, convective and diffusive transport compete with each other in opposing directions and the advection-diffusion equation is used to describe this:

$$
D\left(\frac{1}{r} \frac{\partial}{\partial r}\left(r \frac{\partial c_{s}}{\partial r}\right)+\frac{\partial^{2} c_{s}}{\partial^{2} z}\right)-u_{z} \cdot \frac{\partial c_{s}}{\partial z}=0
$$


Here $D$ is the solute diffusivity, $c_{s}$ is the molar concentration of solute and $u_{z}$ is the convective velocity along the z-direction. A full model should account for the radial variation of the velocity as well but it is assumed that this decays fast and, due to the high aspect ratio of the pore, the problem becomes rapidly one-dimensional (this may be shown to also be true for the concentration field, though radial effects were nonetheless included in the numerical implementation). The following boundary conditions are used to solve the above equation. Along the radial axis, a no-flux boundary condition was imposed along the plane of symmetry and at the pore-feed interface. At the pore-feed interface, $\mathrm{c}_{\mathrm{s}}$ was set equal to $\mathrm{c}_{\mathrm{sF}}$. To calculate $\mathrm{u}_{\mathrm{z}}$ in the above equation, the integrated mass flux is averaged over the pore-film interface and converted to a velocity:

$$
u_{z}=\frac{1}{A_{p}} \frac{M_{w}}{\rho_{w}} D_{w} \int_{\substack{\text { film-pore } \\ \text { interface }}} \frac{d c_{w}}{d z}
$$

where $A_{p}$ is the cross-sectional area of the pore.

A no-flux boundary condition was set at the film-pore interface and at the pore-support interface in both the radial (r) and axial (z) directions.

\subsection{Computational details}

Calculations were made with different structural metrics varied as follows: pore radius was varied over two orders of magnitude from $0.01 \mu \mathrm{m}$ to $0.1 \mu \mathrm{m}$ to $1 \mu \mathrm{m}$. Pore radii between 0.01 and $0.1 \mu \mathrm{m}$ are estimated to be typical size range in phase inversion cast supports [21]. Sizes between 0.1 and $1 \mu \mathrm{m}$ are typical in newer, more novel supports such as electrospun supports [22]. For porosity, calculated as

$\mathrm{R}_{\mathrm{p}}{ }^{2} / \mathrm{R}_{\mathrm{s}}{ }^{2}$ in Fig.1, values of 45,65 and $85 \%$ were chosen with values around $45 \%$ being typical of phase inversion cast supports [23], porosities around 65\% being reported for some of the newer EO membranes [24]. Electrospun supports commonly have porosities upwards of $85 \%$ [22]. Two thicknesses of 25 and 50 $\mu \mathrm{m}$ were explored: $50 \mu \mathrm{m}$ is the average thickness of the HTI-CTA membrane which, for long, was the 
only FO membrane commercially available from Hydration Technology Innovations. Newer EO membrane supports are constantly pushing the lower limits of thicknesses, with supports as thin as 8-15 $\mu \mathrm{m}$ being fabricated $[22,24]$. In varying solution concentrations (the feed and draw solute were both always $\mathrm{NaCl}$ ), feeds of $0.1,0.5,1$ and $2 \mathrm{M}$ were chosen, representing brackish water, seawater, brine from seawater desalination at $50 \%$ recovery and brine from seawater desalination at $75 \%$ recovery. The draw solution was varied between $0.5,1,2$ and 4M (4M representing an arbitrary maximum concentration). In the calculations, one parameter was changed at a time, for instance, when pore radius was varied porosity and thickness were kept constant by adjusting the model geometry accordingly. The model equations were solved using the commercial finite-element package, COMSOL Multiphysics, version 4.3a. The geometry in Fig. 1 was meshed using triangular-shaped elements with extra-refinement used at the filmpore interface to reliably resolve sharp concentration gradients at this boundary, where rapid variations are expected due to the change in boundary condition from a no-penetration region to a perfect sink.

\section{Results and Discussion}

\subsection{Validation of model with experimental data}

In order to test the accuracy of the developed model, a comparison is made between calculated predictions to experimental results obtained from a model TFC membrane [6]. Parameters used in the simulations were matched with that of the actual membrane: the pore radius was set at $0.2 \mu \mathrm{m}$, porosity was $13 \%$, while film and support thickness were $100 \mathrm{~nm}$ and $20 \mu \mathrm{m}$, respectively. The feed was DI water and draw concentrations of $0.1,0.5,1$ and $1.5 \mathrm{M}$ were tested. The osmotic flux measurements were at a temperature of $20^{\circ} \mathrm{C}$ and cross-flow velocity of $0.26 \mathrm{~m} / \mathrm{s}$, corresponding with a Reynolds number of 1190 [6]. A comparison of experimental and simulated water fluxes is shown in Fig. 2.

\section{FIGURE 2}


The theoretical and experimental fluxes follow a similar trend, with the simulation predicting a lower flux at $0.1 \mathrm{M}$ draw and thereafter predicting higher water fluxes. Deviation from the theoretical flux is greatest at the highest draw solution concentration tested $(1.5 \mathrm{M})$, most likely due to salt leakage through the membrane, which is not accounted for in the model. Note that with a $0.1 \mathrm{M}$ draw, the experimental water fluxes were too low to be reliably measured. A main source for the discrepancy between the model calculations and experimental data may be attributed to solute transport - the polyamide layer of the model TFC membrane is not perfectly selective (as was assumed in the simulations) - and as the water flux increases the salt flux increases as well, since both are proportional to the salt concentration across the thin film. This solute transport presents an additional loss of available driving force not accounted for in the model. Nevertheless, this comparison serves as a satisfactory validation of the developed mathematical model. A future extension of the present work could include the concurrent transport of solvent and solute through the composite.

\subsection{Film and Pore Transport Profiles}

We now turn to examine the model calculations of the concentration distributions of water (in the film) and salt (in the pore), and the resulting transport, as affected by changes in bulk solution concentrations. Fig. 3 shows intensity maps depicting the concentration of water within the film in FO mode (i.e. the support side facing the draw solution). In this set of simulations, the feed concentration was fixed at $0.1 \mathrm{M}$ and draw concentrations were varied from $0.5-4.0 \mathrm{M}$. The color legend corresponds to the molar concentration of water. As the draw concentration was increased, so did the water flux. Further, the flux is seen to be highest at the center of the pore (see Supplemental Fig. 1) where it is undisturbed by the presence of the impermeable support material.

FIGURE 3 
Fig. $4 \mathrm{a}$ and $4 \mathrm{~b}$ depict the solute concentration field within the pore, for FO and PRO mode, respectively. For FO (Fig 4a), the feed concentrations were varied from $0.1-2.0 \mathrm{M}$, while the draw concentration was fixed at 4.0 M. For PRO (Fig 4b), draw concentrations were varied from $0.5-4.0 \mathrm{M}$ while the feed concentration was fixed at $0.1 \mathrm{M}$. In both cases, the pore radius was $0.1 \mu \mathrm{m}$, porosity was $65 \%$ and support thickness was $50 \mu \mathrm{m}$. From Fig. $4 \mathrm{a}$ it is seen that as the feed concentration is increased the severity of dilutive ICP decreases due to the reduction in driving force and hence, water flux. A progressively larger fraction of the pore was seen to be at equilibrium with the bulk draw concentration as the feed increased from 0.1 to 2 M. Similarly, for the PRO mode, it can be seen from Fig. $4 \mathrm{~b}$ that an increase in water flux with an increase in draw concentration leads to more severe ICP.

\section{FIGURE 4}

\subsection{Effect of Pore Radius}

The effect of pore radius on transport is shown in Fig. 5 where 5a represents the severity of ICP in terms of an ICP modulus, here defined as

$$
\text { ICP modulus }=\frac{c_{s, f-s}}{c_{s, D(F)}}
$$

Here, the numerator corresponds to the solute concentration at the film-pore interface and the denominator corresponds to the solute concentration at the either the pore-draw or pore-feed interface depending on whether it is FO or PRO mode, respectively. Fig. 5b translates the severity of ICP into a \%loss in driving force as a result of polarization phenomena in the support structure. Firstly, Fig. 5a seems to imply that concentration of the dilute feed in PRO mode results in perhaps more severe polarization than dilution of the concentrated draw in FO mode. However, when these ICP moduli are translated into a \% loss in driving force across the support layer (due to ICP) it can be seen that FO mode ICP is far more severe than that in PRO mode. In the case of PRO mode, advective flow in the support is directed opposite the solute flux and so confines the solute to the selective-support layer interface, 
creating concentrative polarization; conversely, in FO mode, the advective transport results in a 'stretching' of the dilutive polarization of solute within the support. The severity of ICP is then dictated by how effectively the diffusive transport of solute counters the advective flow and this of course is hindered by the support structure. This severity is greater in the FO mode compared with that in the PRO mode implying that the impact on the net driving force caused by dilution of the concentrated draw stream in the former is far greater than concentration of the diluted feed stream in the latter.

\section{FIGURE 5}

Overall, the difference in performance was insignificant upon increasing the pore radius from 0.01 to $0.1 \mu \mathrm{m}$, but upon further increasing to $1 \mu \mathrm{m}$, a small yet noticeable improvement in performance was seen for both modes. This improvement in performance upon increasing pore radius is illustrated through the water flux (in velocity units), as shown in Fig. 6. The simulation conditions were the same as in Fig. 5. The water flux is sensitive to the available cross-section (quadratic with the diameter), and so reduces considerably with increased pore size. The drop in flux is sharper upon going from 0.1 to $1 \mu \mathrm{m}$ than going from 0.01 to $0.1 \mu \mathrm{m}$. In all three pore sizes, the driving force is the same but the changes in flux, and consequently velocity, imply that the film exhibits a 'constricting' effect on water transport, produced by the presence of the impermeable support, with this effect varying non-linearly with pore radius. Fig. 7 presents a graphical illustration of the effect of flux on ICP in the presence of varying degrees of pore confinement. In this schematic, the three membranes have different pore radius and the porosity is kept constant by increasing the pore-to-pore spacing. The membranes are all subject to the same driving force. The illustration is shown for the PRO mode and the hypothesis is similar for FO mode. In smaller pores the higher velocities carry along more solute molecules to the pore-film interface where they are then distributed over a smaller interfacial area compared to the larger pores. This directly corresponds to a more severe ICP in smaller pores. It is noted that a possible effect of diffusive hindrance at the pore entrance and within the pore itself, is not taken into account here. However, the size ratio 
between the hydrated ions considered here and the typical pore sizes encountered in practice is, in general, much less than 0.1 , which means that hindrance effects may be quite reasonably ignored [25].

FIGURE 6

\section{FIGURE 7}

\subsection{Effect of Porosity}

Fig. 8 demonstrates the effect of porosity on pore transport. Here, feed and draw were $0.1 \mathrm{M}$ and 4.0 $\mathrm{M} \mathrm{NaCl}$, respectively, pore radius was $0.1 \mu \mathrm{m}$ and support thickness was $50 \mu \mathrm{m}$. It was found from Fig. 8a that the variations in porosity produced a moderate improvement in performance, compared with the effect of the pore radius. A similar correlation with $\%$ loss in driving force was observed where ICP severity in FO mode far outweighs that in PRO mode. It was found, again, that the flow rates in the pore were larger for smaller porosities than for the larger porosities implying that both pore radius and porosity affect transport along similar principles, outlined in Fig. 7.

\section{FIGURE 8}

\subsection{Effect of Thickness}

The influence of support thickness on ICP is depicted in Fig. 9. Feed and draw were $0.1 \mathrm{M}$ and 4.0 $\mathrm{M} \mathrm{NaCl}$, respectively, pore radius was $0.1 \mu \mathrm{m}$ and porosity was $65 \%$. Fig. 9a shows a sharp decline in ICP severity as the thickness in decreased by half from 50 to $25 \mu \mathrm{m}$; this relates to a similar dramatic drop in $\%$ driving force lost as seen in Fig. 9b. Driving force losses of around $15 \%$ in the FO mode were among the lowest values observed in this study among all parameters studied. Such an enhancement in performance with reduction in support thickness can be attributed to the fact that the distance over which diffusion occurs, before better mixing is available (through convective transport by the crossflow), becomes shorter. 


\section{FIGURE 9}

\subsection{Relative Influence of Porosity vs. Thickness}

In this study, of the three parameters affecting the value of $S_{\text {int }}$ (Eqn. 1), tortuosity was held constant at unity while porosity and thickness were both doubled and halved, respectively. As seen in the previous section, a reduced thickness seemed to show the greatest increase in performance compared to either increasing pore radius or porosity. This finding has, in fact, been previously experimentally observed by McCutcheon and Bui [22] in work on electrospun nanofiber-supported EO membranes. In order to reliably evaluate our observation, the intrinsic structural parameters were kept constant between two sets of simulations in which porosity and thickness were increased and decreased, by $89 \%$ respectively. One parameter was kept constant when the other one was varied. Thickness was fixed at 50 $\mu \mathrm{m}$ when porosity was increased from 45 to $85 \%$ (corresponding to intrinsic structural parameters of 111 and $59 \mu \mathrm{m}$, respectively) and porosity was kept constant at $65 \%$ when thickness was decreased from 72 to $38 \mu \mathrm{m}$ (corresponding to intrinsic structural parameters of 111 and $59 \mu \mathrm{m}$, respectively). Fig. 10 summarizes the results for the FO (Fig. 10a) and PRO (Fig. 10b) modes. It can be seen that, in both modes, reducing the thickness showed a more significant enhancement in performance compared to that obtained by increasing the porosity. The direct reduction in the path length for solute back diffusion upon decreasing the thickness seemed to far outweigh improvements in performance as a result of increasing porosity. Further, at the intrinsic structural parameter value of $111 \mu \mathrm{m}$, it is seen that \%loss in driving force, mostly only in the FO mode, is slightly different between porosity-tortuosity values of $45 \%-50 \mu \mathrm{m}$ and 65\%-72 $\mu \mathrm{m}$. This finding clearly illustrates that membrane structural contribution to transport resistance cannot be quantified by a "bulk" value, as is commonly done when using the structural parameter as a metric, rather the relative influence of the geometrical parameters play an important role in determining the overall transport behavior.

FIGURE 10 


\subsection{Effect of Varying Draw and Feed Concentrations}

Fig. 11 shows the effect of varying draw and feed concentrations on pore transport in FO (Fig. 11a) and PRO (Fig. 11b) modes. For the sake of brevity, only changes in performance as a function of pore radius are shown here. However, similar trends were observed upon changing solution concentrations while varying porosity and thickness as well. It was seen, from Fig. 11a, that upon increasing the draw concentration and hence water flux, the $\%$ driving force lost increased as well.

\section{FIGURE 11}

\subsection{Sensitivity Analysis}

To determine the influence of the assumption of a water permeance $(A)$ value on the simulation outcome, a sensitivity analysis was performed where the $A$ value was changed between $1.7 * 10^{-12}$ and $3.7 * 10^{-12} \mathrm{~m} / \mathrm{s} / \mathrm{Pa}$ with the median value $\left(2.7 * 10^{-12}\right)$ being our assumption in all the other simulations. The results of this analysis are presented in Fig. 12 where the influence of changing $A$ on $\%$ driving force lost is shown. The FO mode simulations seem to be more sensitive to changes in $A$ than their PRO mode counterparts. This is due to the fact that FO mode ICP is typically more pronounced than PRO mode ICP, especially in the absence of a reverse salt flux (possible only in membranes with solute rejection less than 100\%). While the developed model was seen to be moderately sensitive to changes in $A$ value in the FO

mode it was seen that the different $A$ values did not affect the overall trends outlined in sections $3.3,3.4$ and 3.5. This relaxes the possible influence on model results introduced by the use of the experimentally measured permeance in the pore-film interfacial flux expression, Eq. (4). Further, the findings presented in this study are mainly illustrative of physical trends and are not intended to provide any predictive capacity. Rather, they serve as an illustration of the impact of geometry plays in determining the overall performance of a composite membrane, primarily where film and pore transport are coupled.

\section{FIGURE 12}




\section{Concluding Remarks}

The effect of support layer structural metrics on composite membrane transport was examined using a $2 \mathrm{D}$ numerical model that describes coupled transport in the film and pore regions of a model EO membrane. The study provides insight into the influence of different structural metrics on membrane transport. Flux performance is largely affected by ICP and thus efforts to improve membrane structure should focus on effective ways of mitigating this detrimental phenomenon. This was seen to be best done by decreasing support thickness over either increasing porosity or support pore radius. It was clearly seen that membrane structural contribution to transport resistance is not merely determined by an average of the "bulk" metrics (viz. porosity, tortuosity and thickness), as is commonly done when using the structural parameter as a metric, rather the relative influence of the geometrical parameters play an important role in determining the overall transport behavior. The results also indicate that there is a need to look beyond the traditional optimization of thickness, tortuosity and porosity as even parameters like support pore radius, which do not feature in the intrinsic structural parameter formula, seem to affect performance to a non-insignificant degree. Future osmotic membrane designers could benefit from these findings when weighing the effects of manipulating one parameter versus another.

\section{Acknowledgments}

The authors acknowledge financial support from the National Science Foundation (CBET0933553, CBET) and the U.S. Environmental Protection Agency (\#R834872).

\section{Nomenclature:}

A - permeance

$A_{p}$ - pore area

$\mathrm{c}$ - concentration

$\mathrm{D}$ - diffusivity of solute in water

$\mathrm{D}_{\mathrm{w}}$ - diffusivity of water in the thin-film

f-s - film-support interface

$\mathrm{M}_{\mathrm{H} 2 \mathrm{O}}-$ mol. wt. of water

$\mathrm{r}$ - radial coordinate

$\mathrm{t}_{\mathrm{f}}-$ thin-film thickness 
$\mathrm{u}_{\mathrm{z}}$ - axial velocity component in the pore

$\mathrm{z}$ - axial coordinate

\section{Greek letters:}

$\pi$ - osmotic pressure

$\rho_{\mathrm{H} 2 \mathrm{O}}-$ density of water

\section{Subscripts:}

D - draw

F - feed

s- solute

w - water

\section{References}

[1] T.Y. Cath, A.E. Childress, M. Elimelech, Forward osmosis: principles, applications, and recent developments, Journal of membrane science, 281 (2006) 70-87.

[2] A. Achilli, A.E. Childress, Pressure retarded osmosis: From the vision of Sidney Loeb to the first prototype installation-Review, Desalination, 261 (2010) 205-211.

[3] F. Helfer, C. Lemckert, Y.G. Anissimov, Osmotic power with pressure retarded osmosis: theory, performance and trends-a review, Journal of membrane science, 453 (2014) 337-358.

[4] D. Cohen, C. Ross, Mixing moves osmosis technology forward, Chemical Processing, 67 (2004) 2932.

[5] J.R. McCutcheon, M. Elimelech, Influence of concentrative and dilutive internal concentration polarization on flux behavior in forward osmosis, Journal of membrane science, 284 (2006) 237-247.

[6] S.S. Manickam, J.R. McCutcheon, Model thin film composite membranes for forward osmosis: Demonstrating the inaccuracy of existing structural parameter models, Journal of membrane science, 483 (2015) 70-74.

[7] D.L. Shaffer, J.R. Werber, H. Jaramillo, S. Lin, M. Elimelech, Forward osmosis: Where are we now?, Desalination, 356 (2015) 271-284.

[8] T.Y. Cath, M. Elimelech, J.R. McCutcheon, R.L. McGinnis, A. Achilli, D. Anastasio, A.R. Brady, A.E. Childress, I.V. Farr, N.T. Hancock, Standard methodology for evaluating membrane performance in osmotically driven membrane processes, Desalination, 312 (2013) 31-38.

[9] M.C.Y. Wong, K. Martinez, G.Z. Ramon, E.M.V. Hoek, Impacts of operating conditions and solution chemistry on osmotic membrane structure and performance, Desalination, 287 (2012) 340-349.

[10] G.Z. Ramon, M.C.Y. Wong, E.M.V. Hoek, Transport through composite membrane, part 1: Is there an optimal support membrane?, Journal of membrane science, 415 (2012) 298-305.

[11] G.Z. Ramon, E.M.V. Hoek, Transport through composite membranes, part 2 : Impacts of roughness on permeability and fouling, Journal of membrane science (2013).

[12] J.G. Wijmans, P. Hao, Influence of the porous support on diffusion in composite membranes, Journal of membrane science 494 (2015) 78-85.

[13] M. Bruna, S.J. Chapman, G.Z. Ramon, The effective flux through a thin-film composite membrane, EPL (Europhysics Lett.) 110 (2015) 40005.

[14] M.C.Y. Wong, L. Lin, O. Coronell, E.M.V. Hoek, G.Z. Ramon, Impact of liquid-filled voids within the active layer on transport through thin-film composite membranes, Journal of membrane science 500 (2016) 124-135.

[15] L. Zhu, W. Jia, M. Kattula, K. Ponnuru, E.P. Furlani, H. Lin, Effect of porous supports on the 
permeance of thin film composite membranes: Part I. Track-etched polycarbonate supports, Journal of membrane science (2015) 1-12.

[16] M.A. Frommer, J.S. Murday, R.M. Messalem, Solubility and diffusivity of water and of salts in an aromatic polyamide film, European Polymer Journal, 9 (1973) 367-373.

[17] R.J. Hernandez, R. Gavara, Sorption and transport of water in nylon-6 films, Journal of Polymer Science Part B: Polymer Physics, 32 (1994) 2367-2374.

[18] L.T. Lim, I.J. Britt, M.A. Tung, Sorption and transport of water vapor in nylon 6, 6 film, Journal of Applied Polymer Science, 71 (1999) 197-206.

[19] X. Zhang, D.G. Cahill, O. Coronell, B.J. Mariñas, Partitioning of salt ions in FT30 reverse osmosis membranes, Applied Physics Letters, 91 (2007) 181904.

[20] X. Zhang, D.G. Cahill, O. Coronell, B.J. Mariñas, Absorption of water in the active layer of reverse osmosis membranes, Journal of membrane science, 331 (2009) 143-151.

[21] A.K. Ghosh, E.M.V. Hoek, Impacts of support membrane structure and chemistry on polyamidepolysulfone interfacial composite membranes, Journal of membrane science, 336 (2009) 140-148.

[22] J. McCutcheon, N.-N. Bui, Forward Osmosis, in: BOOK Chapter in "Desalination: Water from Water", John Wiley \& Sons, Inc., 2014, pp. 255-285.

[23] S.S. Manickam, J. Gelb, J.R. McCutcheon, Pore structure characterization of asymmetric membranes: Non-destructive characterization of porosity and tortuosity, Journal of membrane science, 454 (2014) 549-554.

[24] L. Huang, N.-N. Bui, M.T. Meyering, T.J. Hamlin, J.R. McCutcheon, Novel hydrophilic nylon 6, 6 microfiltration membrane supported thin film composite membranes for engineered osmosis, Journal of membrane science, 437 (2013) 141-149.

[25] W.M. Deen, Hindered Transport of Large Molecules in Liquid-Filled Pores, AIChE J. 33 (1987) $1409-1425$. 


\section{Figures}

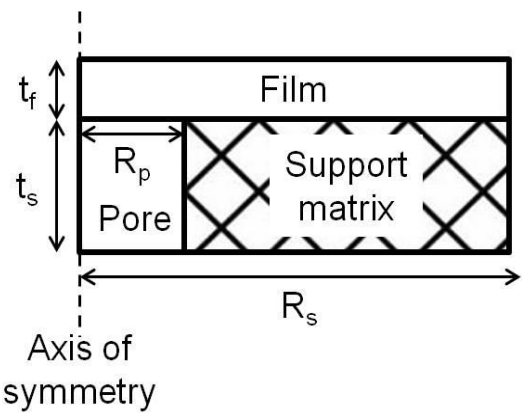

Fig. 1. Schematic drawing illustrating the side view of the 2D cell geometry used for the model calculations. 


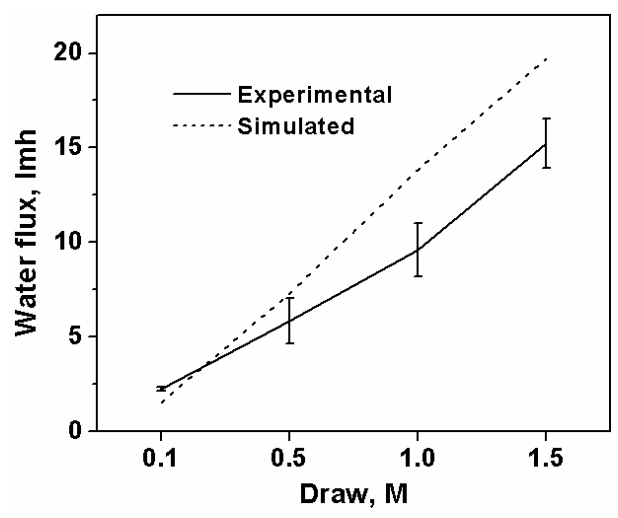

Fig. 2. Validation of the developed model with experimental data (FO mode) from [6]. Simulation conditions matched the experiments with a pore radius of $0.2 \mu \mathrm{m}$ and a porosity of $13 \%$. The film was $100 \mathrm{~nm}$ thick and the support thickness was $20 \mu \mathrm{m}$. The feed was DI water. In the osmotic flux tests, temperature was set at $20^{\circ} \mathrm{C}$ and the cross-flow velocity was $0.26 \mathrm{~m} / \mathrm{s} . \mathrm{N}_{\mathrm{Re}}$ of the test cell channel was $1190[6]$. 


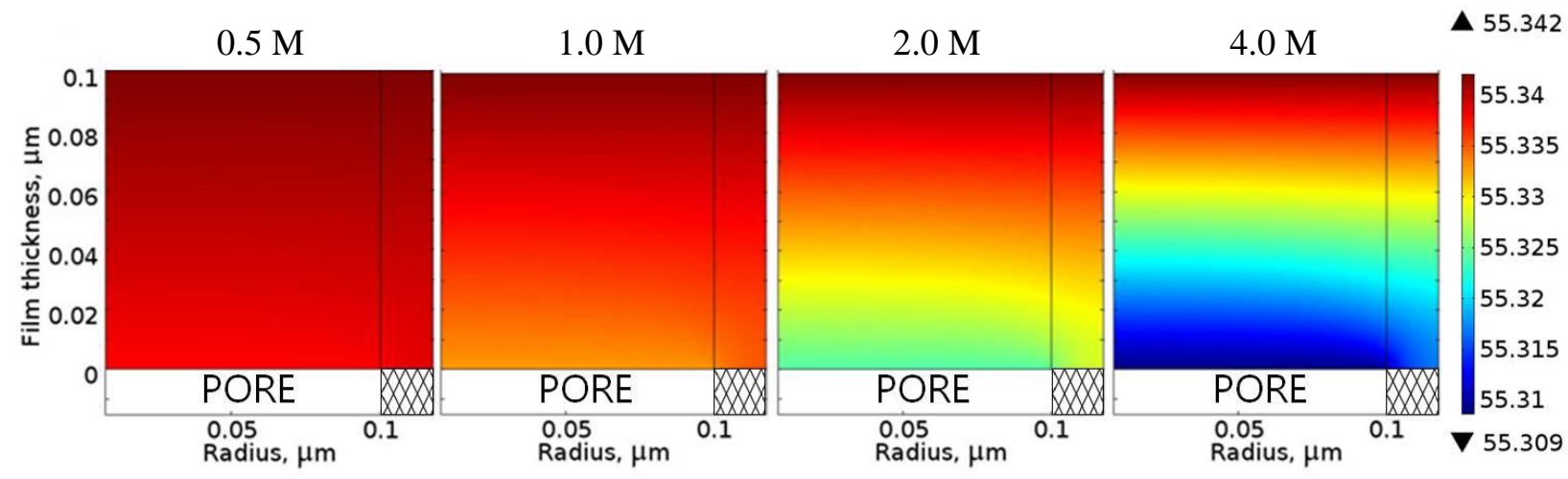

Fig. 3. Intensity maps depicting transport of water through the film in the FO mode. More water is transported as draw concentration is increased, from $0.5-4.0 \mathrm{M}$ to the right. Feed concentration was fixed at $0.1 \mathrm{M}$. The color legend corresponds to the concentration of water in $\mathrm{mol} / \mathrm{l}$. 

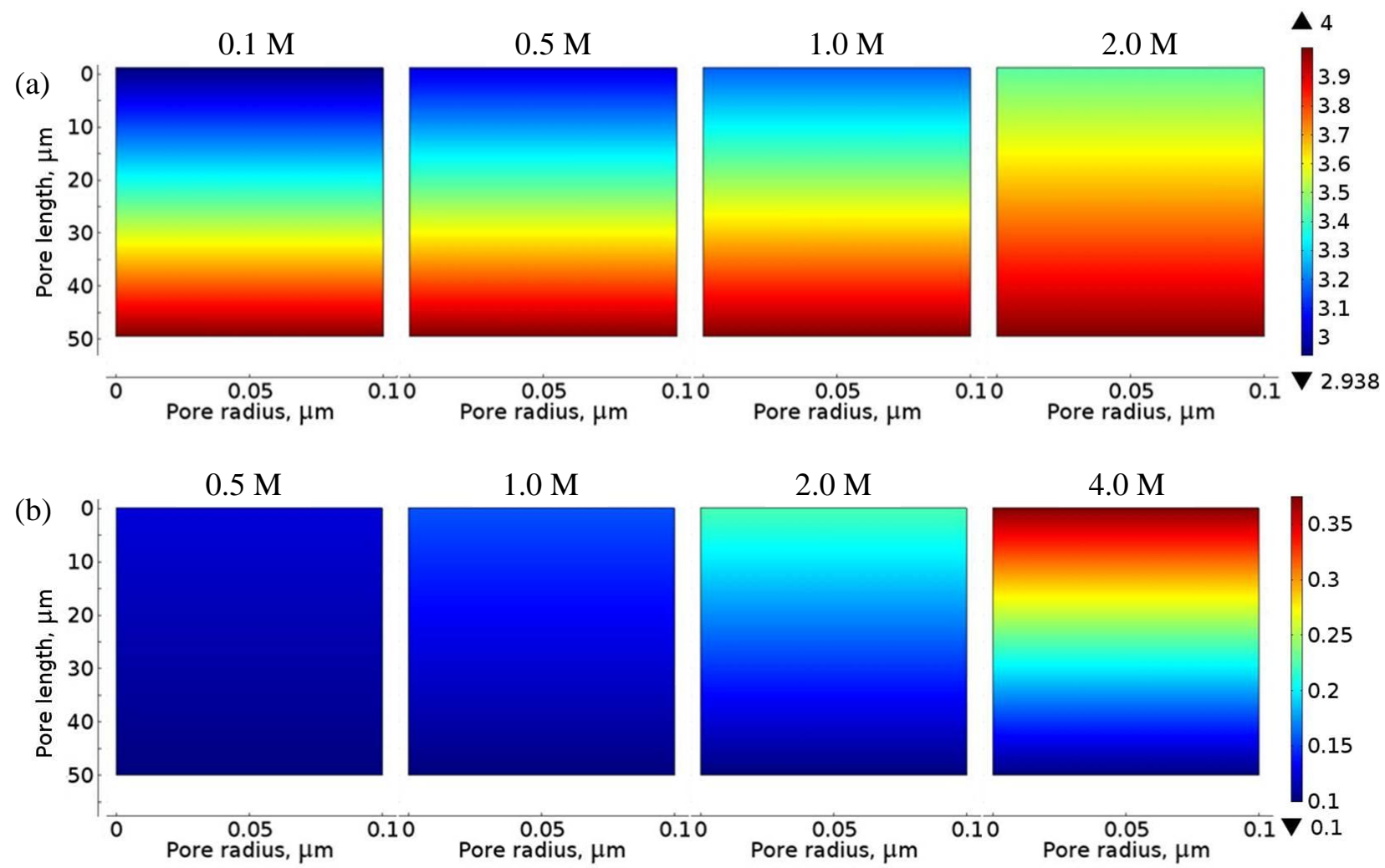

Fig. 4. Intensity maps depicting solute $(\mathrm{NaCl})$ concentration profiles in the pore for $\mathrm{FO}$ mode (a) and PRO mode (b). (a) Severity of dilutive ICP is seen to decrease as feed concentration increases (water flux decreases) from $0.1-2.0 \mathrm{M}$ to the right. Draw concentration was fixed at 4.0 M. (b) Severity of concentrative ICP is seen to increase as draw concentration increases (water flux increases) from $0.5-4.0$ $\mathrm{M}$ to the right. Feed concentration was fixed at $0.1 \mathrm{M}$. $\mathrm{R}_{\mathrm{p}}=0.1 \mu \mathrm{m}, \varepsilon=65 \%$ and $\mathrm{t}_{\mathrm{s}}=50 \mu \mathrm{m}$ in both $4 \mathrm{a}$ and $\mathrm{b}$. The color legend corresponds to the concentration of $\mathrm{NaCl}$ in $\mathrm{mol} / \mathrm{l}$. 

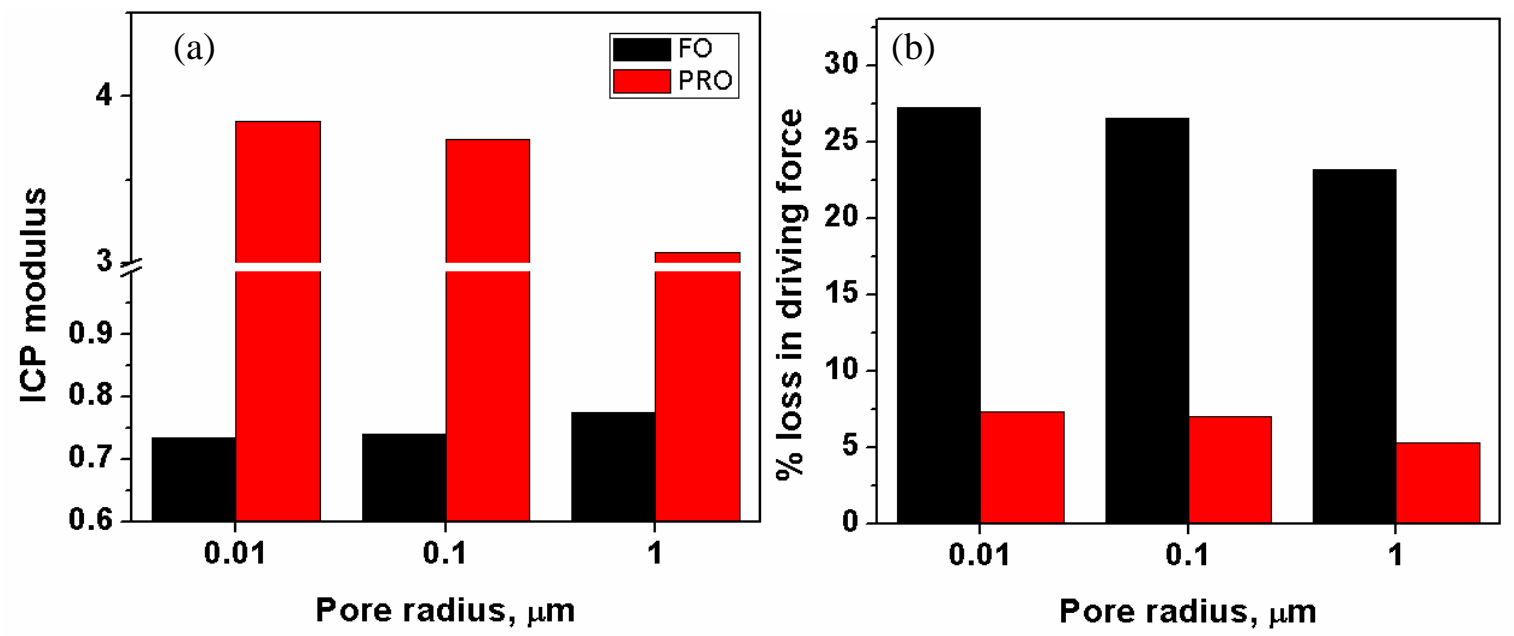

Fig. 5. (a) Effect of pore radius on severity of ICP for FO and PRO modes. (b) \% driving force lost across the support layer due to ICP. Feed and draw were $0.1 \mathrm{M}$ and $4.0 \mathrm{M} \mathrm{NaCl}$, respectively. $\varepsilon=65 \%$ and $\mathrm{t}_{\mathrm{s}}=$ $50 \mu \mathrm{m}$. 


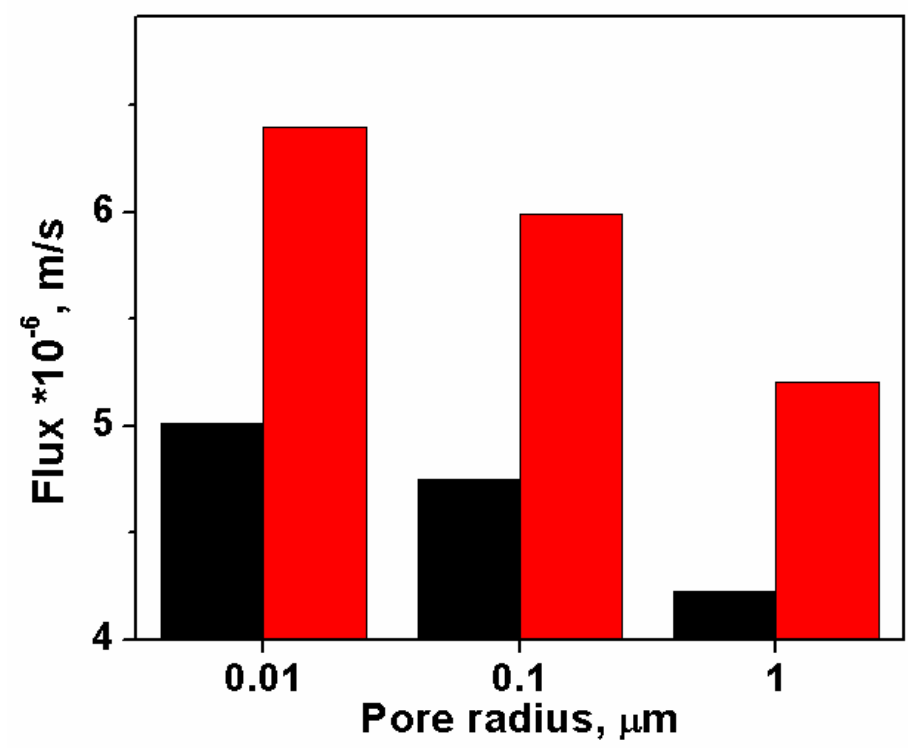

Fig. 6. Flux (in velocity units; $1 \mu \mathrm{m} / \mathrm{s}=3.6 \mathrm{Lm}^{-2} \mathrm{hr}^{-1}$ ) as a function of pore radius. Feed and draw were 0.1 $\mathrm{M}$ and $4.0 \mathrm{M} \mathrm{NaCl}$, respectively. $\varepsilon=65 \%$ and $\mathrm{t}_{\mathrm{s}}=50 \mu \mathrm{m}$. 

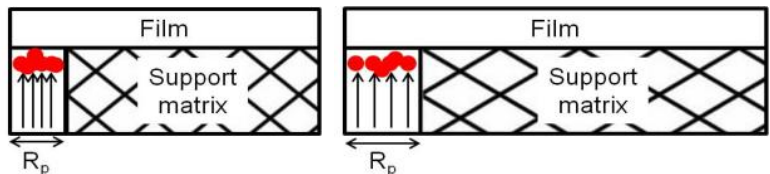

$\underset{\mathrm{R}_{\mathrm{p}}}{\stackrel{\rightleftarrows}{\rightleftarrows}}$

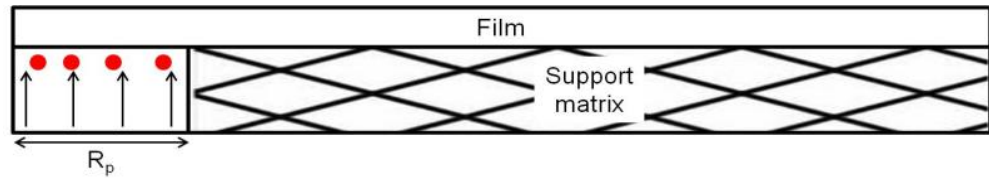

- $\mathrm{NaCl}$

Fig. 7. Schematic showing the hypothetical effect of pore radius on severity of ICP, using the PRO mode for illustration. Note that all three membranes are subjected to the same driving force, i.e. similar draw and feed concentrations. For smaller pore sizes, higher convective flux (indicated by arrows) carries along more solute molecules to the pore-film interface which are then subsequently distributed over a smaller area thus leading to more severe ICP. 

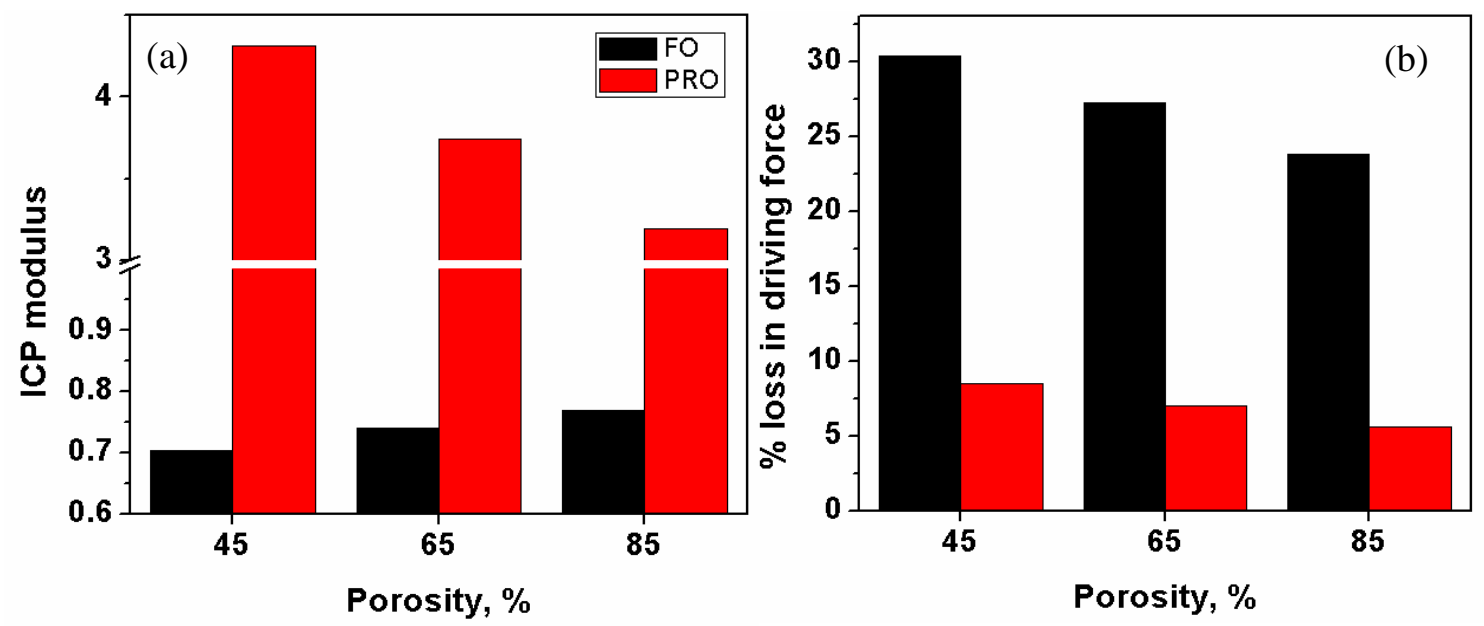

Fig. 8. (a) Effect of porosity on severity of ICP for FO and PRO modes. (b) \% driving force lost across the support layer due to ICP with FO mode losses being much more severe than PRO mode ones. Feed and draw were $0.1 \mathrm{M}$ and $4.0 \mathrm{M} \mathrm{NaCl}$, respectively. $\mathrm{R}_{\mathrm{p}}=0.1 \mu \mathrm{m}$ and $\mathrm{t}_{\mathrm{s}}=50 \mu \mathrm{m}$. 


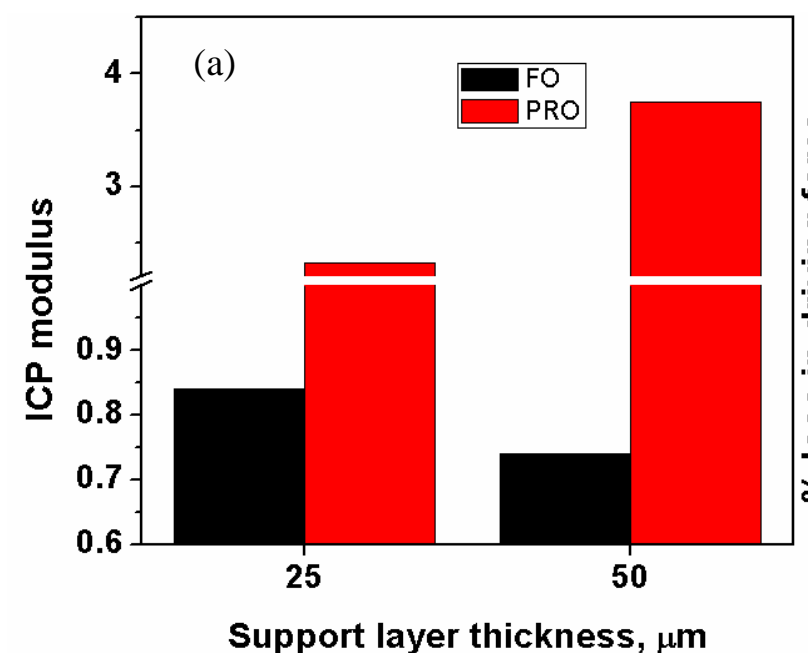

Support layer thickness, $\mu \mathrm{m}$

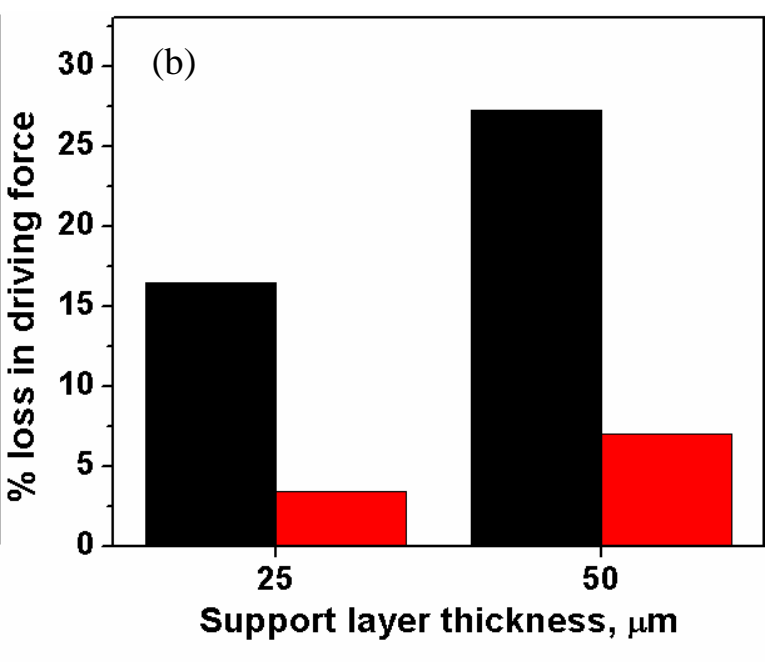

Fig. 9. (a) Effect of thickness on severity of ICP for FO and PRO modes. (b) \% driving force lost across the support layer due to ICP with FO mode losses being much more severe than PRO mode ones. Feed and draw were $0.1 \mathrm{M}$ and $4.0 \mathrm{M} \mathrm{NaCl}$, respectively. $\mathrm{R}_{\mathrm{p}}=0.1 \mu \mathrm{m}$ and $\varepsilon=65 \%$. 

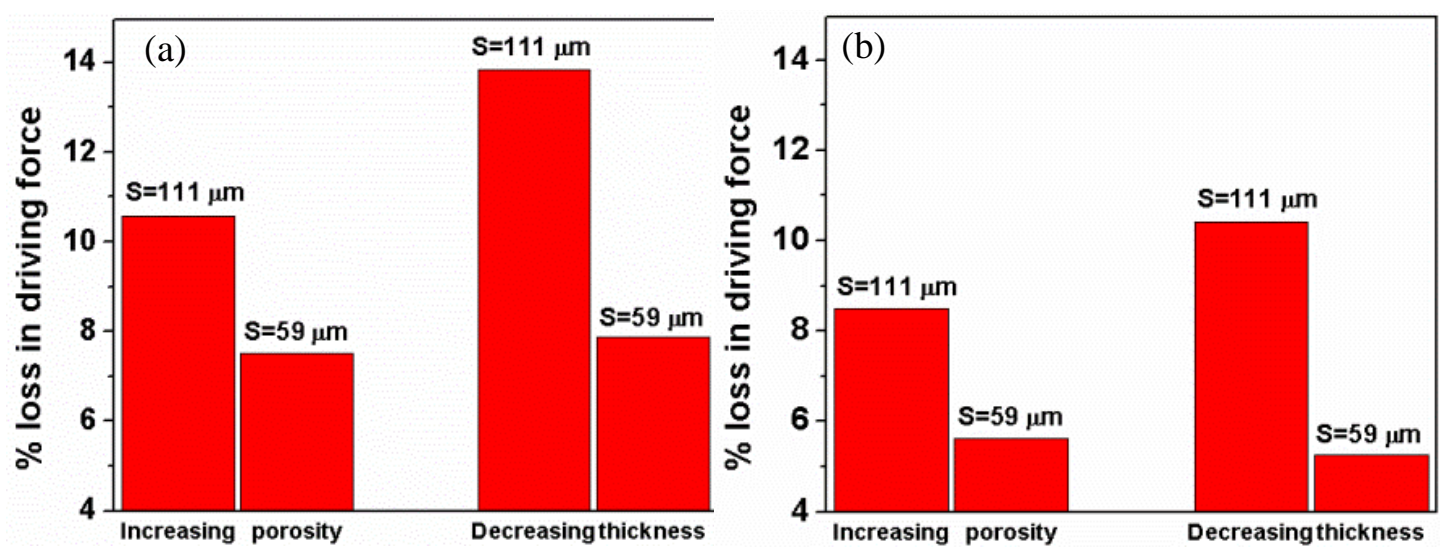

Fig. 10. Relative influences of changing porosity and thickness independently while varying structural parameter between two values, viz. 111 and $59 \mu \mathrm{m}$, in (a) FO and (b) PRO mode. While porosity was increased from 45 to $85 \%$ the thickness was kept constant at $50 \mu \mathrm{m}$ and when thickness was decreased from 72 to $38 \mu \mathrm{m}$ porosity was kept constant at $65 \%$. It is to be noted that both porosity and thickness were changed by $89 \%$. Feed and draw were $0.1 \mathrm{M}$ and $4.0 \mathrm{M} \mathrm{NaCl}$, respectively and pore radius was 0.1 $\mu \mathrm{m}$. 

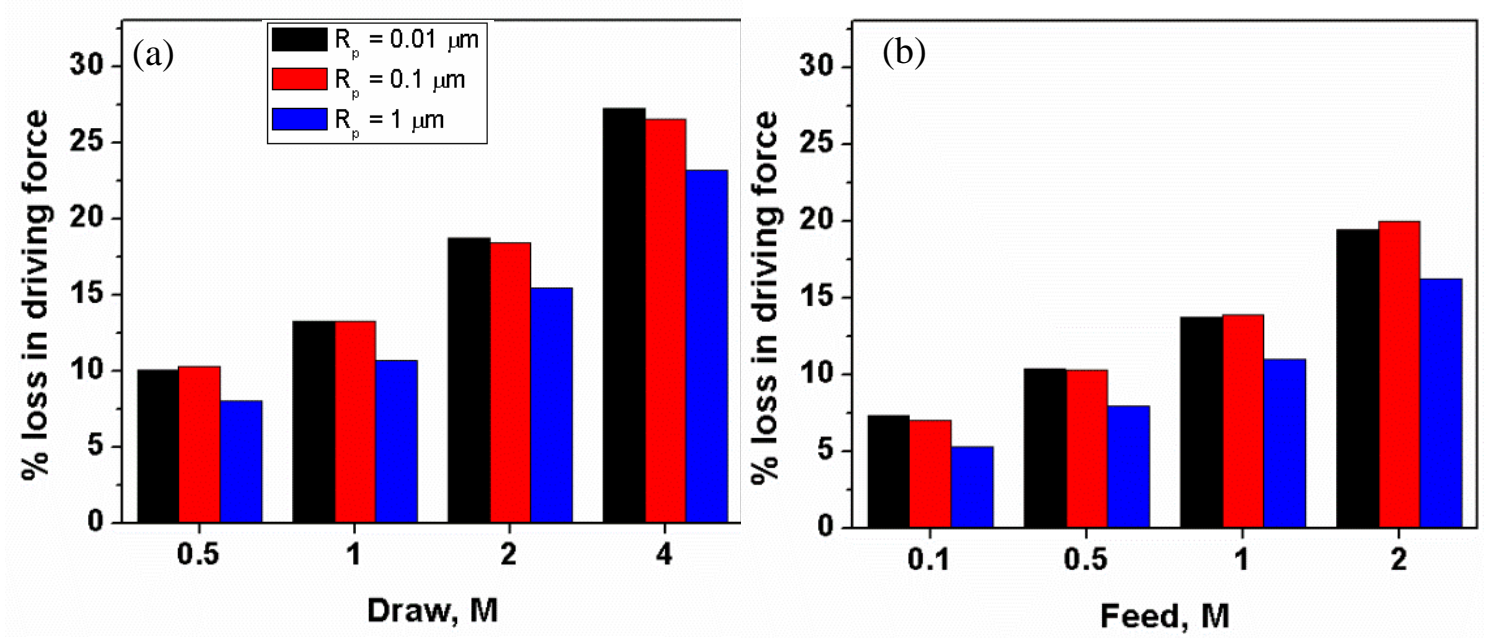

Fig. 11. Effects of varying concentrations of (a) draw in the FO mode and (b) feed in the PRO mode on the severity of ICP, depicted here as a $\%$ loss in driving force, for different pore radii. $\varepsilon=65 \%$ and $\mathrm{t}_{\mathrm{s}}=50$ $\mu \mathrm{m}$. 


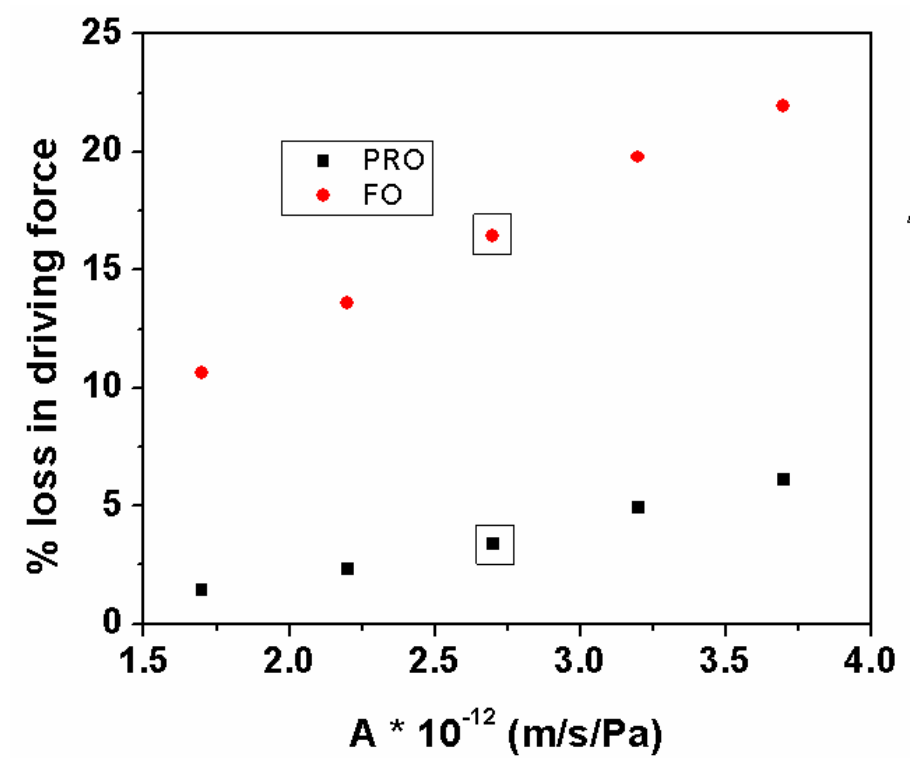

Fig. 12. Analysis of the sensitivity of the developed model to changes in the membrane permeance, A, in both FO and PRO mode. The data points that are highlighted with a square refer to the "base" A value used in all other simulations in this study. The FO mode simulations were more sensitive to changes in A values compared to the PRO mode ones. Feed and draw were $0.1 \mathrm{M}$ and $4.0 \mathrm{M} \mathrm{NaCl}$, respectively. 


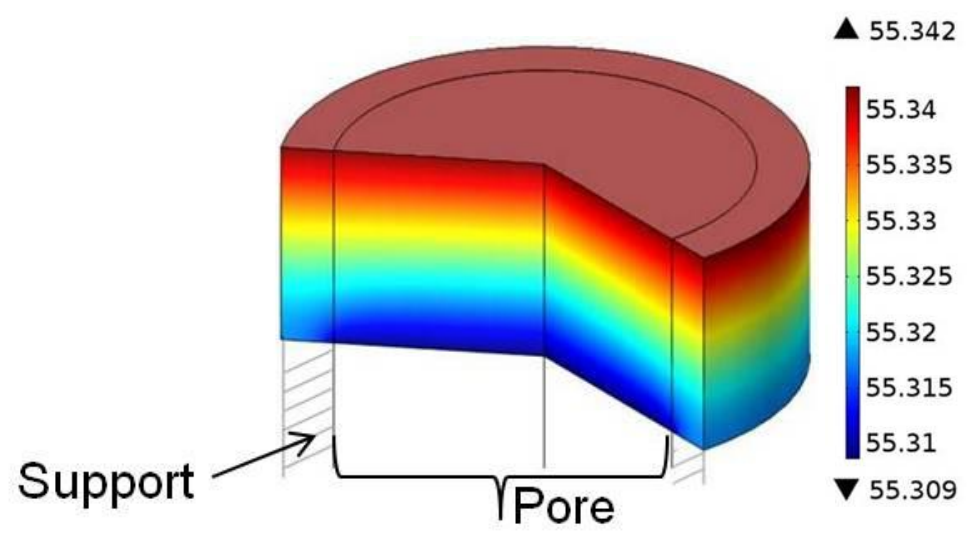

3D intensity map depicting transport of water through the film in the FO mode. The water flux is seen to be greatest along the central axis of the pore and lowering in intensity as it approaches the edges of the pore. The color legend corresponds to the concentration of water in $\mathrm{mol} / \mathrm{l}$. 